\title{
On the Computation of Discrete Logarithms in Class Groups
}

\author{
Extended Abstract
}

\author{
Johannes Buchmann \\ Stephan Düllmann \\ FB14-Informatik \\ Universität des Saarlandes \\ D-6600 Saarbrücken \\ West Germany
}

\section{Introduction}

In [3] and [1] a new key exchange system was introduced whose security is related to the difficulty of solving the discrete logarithm problem in the class group of imaginary quadratic orders. Subsequently, in [5] and [4] a subexponential algorithm for computing those class groups was presented and it was shown how to use this algorithm and the index-calculus method to calculate discrete logarithms.

In this paper we show how the output of the class group algorithm can be used to simplify the index-calculus algorithm in class groups considerably. This simplification enables us to use a slight modification of our implementation [2] of the algorithm of Hafner and McCurley to calculate discrete logarithms in fairly large class groups. At the end of the paper we will present the results of some experiments which show that the computation of discrete logarithms in class groups is very easy once the class group has been computed by the subexponential algorithm. 


\section{The idea}

Let $G$ be a finite abelian group. Let

$$
G=\left\langle\gamma_{1}>\times \ldots \times<\gamma_{l}>\right.
$$

be a presentation of $G$ as a direct product of cyclic groups. Suppose for $\alpha, \beta \in G$ we wish to find $x \in \mathcal{Z}$ with

$$
\alpha^{x}=\beta .
$$

The method we suggest is very simple: Calculate the representations

$$
\alpha=\prod_{i=1}^{l} \gamma_{i}^{a_{i}}, \beta=\prod_{i=1}^{l} \gamma_{i}^{b_{i}}
$$

Then (2) implies

$$
\prod_{i=1}^{l} \gamma_{i}^{z \cdot a_{i}}=\prod_{i=1}^{l} \gamma_{i}^{b_{i}}
$$

which is solvable if and only if the system of simultaneous congruences

$$
x a_{i} \equiv b_{i} \bmod g_{i} \quad(1 \leq i \leq l)
$$

where $g_{i}=\left|\left\langle\gamma_{i}\right\rangle\right|(1 \leq i \leq l)$ has a solution. This system can be solved by means of the generalized chinese remainder theorem.

\section{Application to class groups}

Let $\mathrm{Cl}$ be the class group of an imaginary quadratic order of discriminant $D$. The algorithm of Hafner and McCurley starts from a generating set $\left\{\mathcal{P}_{1}, \ldots, \mathcal{P}_{k}\right\}$ for $C l$ and subsequently calculates a basis $\left\{\underline{b}_{1}, \ldots, \underline{b}_{k}\right\} \subseteq \mathcal{Z}^{k}$ for the relation lattice

$$
L=\left\{\underline{e}=\left(e_{1}, \ldots, e_{k}\right) \in \mathcal{Z}^{k}: \prod_{i=1}^{k} \mathcal{P}_{i}^{e_{i}}=1\right\}
$$


Then the Smith normal form

$$
S=\operatorname{diag}\left(g_{1}, \ldots, g_{l}, 1, \ldots, 1\right) \in \mathcal{Z}^{k \times k}
$$

of $B=\left(\underline{b}_{1}, \ldots, \underline{b}_{k}\right)$ is calculated, $\left(g_{l}>1\right)$,

$$
S=U^{-1} B V
$$

with $U, V \in \mathrm{GL}(k, \mathcal{Z})$. The transformation matrices $U$ and $U^{-1}$ are also output of the algorithm. If we put $U=\left(u_{i j}\right)_{k \times k}$ and

$$
G_{i}=\prod_{j=1}^{k} \mathcal{P}_{j}^{u_{i j}} \quad(1 \leq i \leq l)
$$

then

$$
C l=<G_{1}>\times \ldots \times<G_{l}>
$$

and

$$
\left|<G_{i}>\right|=g_{i} \quad(1 \leq i \leq l) .
$$

Conversely, if we let $U^{-1}=\left(u_{i, j}^{\prime}\right)_{k \times k}$ then we have

$$
\mathcal{P}_{i}=\prod_{j=1}^{l} G_{j}^{u_{i, j}^{\prime}} \quad(1 \leq i \leq k)
$$

Moreover, if we are given a representation of an element in the class group on the generating system $\left\{\mathcal{P}_{1}, \ldots, \mathcal{P}_{k}\right\}$, then we can use (5) to come up with a representation of that element on the generating system $\left\{G_{1} \ldots, G_{l}\right\}$.

Now suppose that we want to solve

$$
A^{x}=B
$$

in $C l$. In order to apply the ideas of the previous section it is sufficient to determine representations

$$
A=\prod_{i=1}^{k} \mathcal{P}_{i}^{a_{i}^{\prime}}, B=\prod_{i=1}^{k} \mathcal{P}_{i}^{b_{i}^{\prime}}
$$


since those can be transformed into presentations

$$
A=\prod_{i=1}^{l} G_{i}^{a_{i}}, B=\prod_{i=1}^{l} G_{i}^{b_{i}} .
$$

But those presentations are computed as follows: Let $p_{1}, \ldots, \wp_{k}$ be first degree prime ideals such that $\mathcal{P}_{i}$ is the equivalence class of $\mathfrak{p}_{i}(1 \leq i \leq k)$ and let $\mathcal{A}$ be the reduced ideal in the class of $A$. For random exponent vectors $\underline{v}=\left(v_{1}, \ldots, v_{k}\right) \in[0, \ldots D-1]^{k}$ we calculate the reduced ideal $\mathcal{A}^{\prime}$ in the class of $\mathcal{A} \prod_{i=1}^{k} \wp_{i}^{v_{i}}$ until we find such an $\mathcal{A}^{\prime}$ which can be written as $\mathcal{A}^{\prime}=\prod_{i=1}^{k} \wp_{i}^{v_{i}^{\prime}}$. Then

$$
\mathcal{A}=\prod_{i=1}^{k} \wp_{i}^{v_{i}^{i}-v_{i}}
$$

is the presentation we were looking for. Analogeously, we can find such a representation for a reduced ideal $\mathcal{B}$ in the class of $B$. If we use all the prime ideals whose norm is bounded by $L[\beta]$ where for $\beta>0$ we use the notation

$$
L[\beta]=(\exp \sqrt{\log D \log \log D})^{\beta+o(1)}
$$

as usual then each trial takes time $L[0]$ and the probability for being able to factor $\mathcal{A}^{\prime}$ is $L\left[-\frac{1}{4 \beta}\right]$. So the expected running time of the procedure that finds the representation on the original generating system takes time $L\left[\frac{1}{4 \beta}\right]$. The computation of the representation on the generating system $\left\{G_{1}, \ldots, G_{l}\right\}$ can be carried out in time $L[\beta]$. The optimal value for $\beta$ is $\beta=\frac{1}{2}$. Hence the expected running time for each new discrete logarithm problem is $L\left[\frac{1}{2}\right]$.

\section{Numerical results}

In the tables below we present the results of some experiments concerning the computation of discrete logarithms in class groups of imaginary quadratic fields. In the first table we give the following data:

- discriminant $D$, 
- size $k$ of the generating $\operatorname{set}\left\{\mathcal{P}_{1}, \ldots, \mathcal{P}_{k}\right\}$,

- size $l$ of the generating system $\left\{G_{i}, \ldots G_{l}\right\}$,

- values of $g_{i}(1 \leq i \leq l)$,

- class number $h$.

\begin{tabular}{|c|c|c|r|l|}
\hline$D$ & $k$ & $l$ & $h$ & $g_{i}(1 \leq i \leq l)$ \\
\hline$-\left(4 \cdot 10^{24}+4\right)$ & 1404 & 3 & 1154987161920 & 2,4, \\
$-\left(4 \cdot 10^{29}+4\right)$ & 1902 & 3 & 436605442139682 & 144373395240 \\
& & & 2,2, \\
$-\left(4 \cdot 10^{34}+4\right)$ & 2609 & 4 & 189652590177168096 & \\
& & & $2,2,2$, \\
$-4 \cdot F_{7}$ & 3257 & 2 & 17787144930223461408 & 23706573772146012 \\
& & & & 8893572465111730704 \\
\hline
\end{tabular}

$\left(4 \cdot F_{7}=4 \cdot\left(2^{2^{7}}+1\right)=1361129467683753853853498429727072845828\right)$

In the second table we present the running times for the several stages of the algorithm. All timings given here are seconds CPU-time on a Sun 4/60-Sparc Station 1.

- time $t_{1}$ to compute the sets $\left\{G_{1}, \ldots, G_{l}\right\},\left\{g_{1}, \ldots, g_{l}\right\}$, the transformation matrices $U$ and $V$ and the class number $h$ using our implementation [2].

- time $t_{2}$ to determine the representations (6) for given $A$ and $B$,

- time $t_{3}$ to transform the representation (6) for given $A$ and $B$ into the presentation (7).

- time $t_{4}$ to solve the simultaneous congruences (3). 
Note that $t_{2}, t_{3}$ and $t_{4}$ are very small compared to $t_{1}$ and so the effort for each new discrete logarithm problem in the same field is quite small.

\begin{tabular}{|c|c|c|c|c|}
\hline$D$ & $t_{1}$ & $t_{2}$ & $t_{3}$ & $t_{4}$ \\
\hline$-\left(4 \cdot 10^{24}+4\right)$ & 3418 & 5 & 4 & $<1$ \\
$-\left(4 \cdot 10^{29}+4\right)$ & 18587 & 21 & 5 & $<1$ \\
$-\left(4 \cdot 10^{34}+4\right)$ & 123404 & 37 & 10 & $<1$ \\
$-4 \cdot F_{7}$ & 511978 & 96 & 18 & $<1$ \\
\hline
\end{tabular}

\section{References}

[1] J. Buchmann, S. Düllmann, H.C. Williams, On the complexity and efficiency of a new key exchange system, Proceedings EUROCRYPT'89, to appear.

[2] J. Buchmann, S. Düllmann, A probabilistic class group and regulator algorithm and its implementation, Proceedings Colloquium on Computational Number Theory, Debrecen 1989, to appear.

[3] J. Buchmann, H.C. Williams, A key exchange system based on imaginary quadratic fields, J. Cryptology 1 (1988), 107-118.

[4] J.L. Hafner, K.S. McCurley, A rigorous subexponential algorithm for computation of class groups, Journal AMS, to appear.

[5] K.S. McCurley, Cryptographic key distribution and computation in class groups, Proceeding of NATO ASI Number Theory and Applications, Kluwer Academic Publishers 1989, 459-479. 